\title{
Gestión de la experiencia de las unidades principales de internacionalización en las instituciones de educación superior
}

Vivian P. Picalúa ${ }^{{ }^{\star}}$, Karen M. Payares ${ }^{1}$, Evaristo J. Navarro ${ }^{1}$ y Kennedy Hurtado ${ }^{2}$

(1) Universidad de la Costa, Departamento de Ciencias Empresariales, Barranquilla, Colombia

(correo-e: vpicalua1@cuc.edu.co; kpayares4@cuc.edu.co; enavarro3@cuc.edu.co).

(2) Universidad del Atlántico, Departamento de Ciencias Básicas, Barranquilla, Colombia

(correo-e: kennedyhurtado@mail.uniatlantico.edu.co).

*Autor a quien debe ser dirigida la correspondencia

Recibido Jun. 24, 2020; Aceptado Ago. 26, 2020; Versión final Oct. 21, 2020, Publicado Abr. 2021

\begin{abstract}
Resumen
El objetivo del presente estudio es analizar la gestión de la experiencia de las unidades principales de internacionalización en las instituciones de educación superior de Barranquilla (Colombia). Se utiliza un enfoque epistemológico deductivo, paradigma cuantitativo de tipo descriptivo y un diseño no experimental de campo. Como técnica se usó la encuesta y se utilizó el cuestionario de escala tipo Likert aplicado a 13 directores pertenecientes a la unidad principal de internacionalización de las instituciones de educación superior que estén activas en el sistema nacional de información de la educación superior. Los datos recolectados fueron procesados a través de la técnica estadística de regresión múltiple. Como resultado se identifica una función matemática y se deduce que el proceso de la experiencia representa la contribución más alta con un $91.5 \%$. Se concluye que la gestión de la experiencia es una herramienta cuando se implementan estrategias, se establecen procesos para la experiencia y se diseña el servicio.

Palabras clave: gestión; experiencia; diseño; servicio; internacionalización; instituciones; educación superior
\end{abstract}

\section{Experience management by the international office of higher education institutions}

\begin{abstract}
The main objective of this research study is to examine the management of customer experience by international offices of higher education institutions located in Barranquilla (Colombia). This study has a deductive epistemological approach, a descriptive quantitative paradigm, and a non-experimental field design. A survey with a Likert scale questionnaire is applied to 13 directors of international offices. Collected data is processed by multiple regression analyses. The results identify a mathematical function and deduce that the customer experience process represents the highest contribution with $91.5 \%$. It is concluded that that experience management is a tool when strategies are implemented, when experience processes are established, and when the service is designed.
\end{abstract}

Keywords: experience management; service design; internationalization; higher education institutions 


\section{INTRODUCCIÓN}

La internacionalización es considerada un punto de referencia en el siglo XXI para el desarrollo de la educación superior. Así mismo, la globalización ha permitido que la economía sea más dinámica principalmente en los procesos comerciales; generando la integración de espacios educativos internacionales mediante la innovación tecnológica; siendo este un espacio democrático, justo e igualatorio para todos; que requiere de aprendizajes orientados a adquirir habilidades y técnicas útiles para garantizar el progreso (Ortega, 2020). Esta evolución ha generado cambios en la orientación del cliente, y por lo tanto la necesidad de integrar el marketing en los servicios ofrecidos por las instituciones de educación superior (IES).

Este proceso de cambio económico y social da como resultado el surgimiento de una nueva manera de ofrecer los productos y servicios en el mercado. En poco tiempo, la experiencia se ha convertido en uno de los conceptos de marketing dominantes para profesionales y académicos (De keiser et al., 2020). Por esta razón, la literatura existente revela posibles razones de inclinación hacia la "experiencia", entre las cuales, la perspectiva del cliente, la orientación global, la necesidad de servicios personalizados, estilos de vida, y avances en la tecnología de las comunicaciones generan una mayor conciencia de las necesidades de los consumidores, brindándoles disponibilidad de diversas alternativas en el mercado (Geetika et al., 2017).

La mentalidad cultural enfatiza la relevancia de una cultura organizacional que ve la experiencia como parte de una red de mercadeo que requiere la captura de datos del cliente más allá de las actitudes sensoriales y respuestas del comportamiento, para llegar a ese valor debe trabajar en capacidades organizativas que mantienen el equilibrio de la organización y estas son: 1) el monitoreo del recorrido del punto de contacto; 2) priorizar los puntos de contacto y 3 ) adaptar los puntos de contacto al diseño del viaje del cliente (Holmlund et al., 2020).

Las anteriores necesidades impulsan el surgimiento de la gestión de la experiencia. Porque, la interacción entre el consumidor y el producto es el corazón de la experiencia real del consumo, y la gestión de la experiencia inicia con el análisis del mundo experiencial y continua la etapa de comprender innovaciones constantes, es decir, es la recopilación de datos experienciales con todos los clientes de la empresa y su relación con la innovación para mejorarlas (Jaziri, 2019). Es así como, las organizaciones deben construir las experiencias con la innovación continua, para que esa interacción dé como resultado diferentes tipos de respuestas que puedan ser analizadas por la organización.

Desde una mirada por América Latina países como Brasil y Chile trabajan orientados en el mantenimiento de las relaciones internacionales, principalmente en la oferta de servicios académicos. Desde el entorno educativo, la declaración mundial de educación de 1998 ha manifestado que es importante la creación de una oficina o dirección de cooperación especializada en la gestión de las relaciones internacionales. En este contexto, es la Unidad Principal de Internacionalización (UPI), siendo esta, la imagen institucional dentro de las instituciones para la implementación de estrategias y el diseño de indicadores enfocados en la mejora de la calidad en el sistema de educación superior. La implementación de acciones de mejoramiento que fortalezcan las relaciones entre los clientes internos y externos, manteniendo la lealtad del cliente a largo plazo, porque introducir la gestión de la experiencia como recurso superior, conlleva a una mentalidad cultural hacia la experiencia del cliente (Andajani, 2015).

Por su parte, el Observatorio Colombiano de Ciencia y Tecnología (OCyT, 2013) afirma que las UPI en Colombia, trabajan en la gestión de la internacionalización de la docencia, investigación y extensión, desde un contexto dinámico para orientar el mantenimiento de las relaciones internacionales, desarrollando el lobbying como herramienta de comunicación y la publicidad, resaltando que se deben trabajar en áreas temáticas que se encuentran principalmente en: 1) asesoramiento por parte del cliente interno; 2) diseño de servicio para la atención; 3) promoción y acompañamiento de las convocatorias; 4) movilidad docente y estudiantil; 5) oportunidades de investigación y 6) convenios internacionales. El incumplimiento de estas prácticas le dificultaría a Colombia lograr ubicarse entre los países más competitivos de América Latina para el año 2032 (Salmi et al., 2014).

Específicamente, las instituciones de la Región Caribe deben animarse a generar un diagnóstico interno detallado del plan de internacionalización a nivel institucional, valiéndose de las ventajas y estrategias que el marketing relacional ofrece para actividades que involucran cooperación y relacionamiento con varios grupos de interés a nivel nacional e internacional (Palacio y Rondón, 2018) y la necesidad del desarrollo permanente de la gestión de la experiencia en su constante avance.

Es así como, la experiencia académica en otros espacios educativos, culturales y sociales permiten hacer seguimiento a las IES de las impresiones de los propios beneficiarios, puesto que las experiencias adquiridas por los estudiantes son un insumo valioso a la hora de evaluar la situación de los programas académicos institucionales, su pertinencia y abordaje respecto a los socios internacionales (Ministerio de Educación 
Nacional, 2015). Por esta razón, se presenta el objetivo de este estudio que consiste en examinar qué aspectos de la gestión de la experiencia son una herramienta para la internacionalización de las IES. Respecto a la estructura de este trabajo, se revisó la literatura, se describió la metodología, se examinó los resultados por el modelo, y se analizó los resultados alcanzados. Finalmente se expresan las discusiones y conclusiones de esta investigación, siendo un aporte para los directores de las UPI de las instituciones de educación superior.

\section{OTROS ANTECEDENTES}

En lo que sigue se presentan conceptos relacionados con la importancia de la internacionalización en las IES y las bases teóricas relacionadas con la gestión de la experiencia con sus factores independientes.

\section{Internacionalización de las instituciones de educación superior}

La internacionalización es considerada como el conjunto de múltiples actividades, programas y servicios que están vinculados a los estudios nacionales para el intercambio educativo y la cooperación técnica en las instituciones, así mismo es definido como un proceso estratégico cuya meta es la integración de la dimensión o perspectiva internacional en las funciones sustantivas de la docencia, investigación y extensión (Aguilar, 2017).Según Jaramillo (2003) comenzar a generar la cultura de internacionalización en las instituciones de educación en Colombia es un paso y un reto fundamental para incursionar en el mundo del conocimiento y del saber, teniendo presente que, para promover este paso, es necesario que se origine la identidad nacional, el mejoramiento de la competitividad económica y la tecnología de la educación en un ámbito internacional desde la educación. Es necesario incorporar nuevos mecanismos que permitan fortalecer las instituciones de educación superior y que fomenten cooperación entre ellas, con la finalidad de proporcionar la formación de capital humano avanzado, y de esta forma, poder enfrentar los desafíos que posee la sociedad (Huerta y Gaete,2019).

Es por esta razón que las UPI pueden desempeñar un rol facilitador y coordinador importante en las instituciones de educación superior. La internacionalización no será sólida sin un equipo diverso de oficinas de todas las instituciones involucradas, independientemente de su forma particular, es decir, que es importante que exista un liderazgo individual que se apropie de las funciones y mejore cada procedimiento. Por consiguiente, las UPI deben desarrollar estrategias que estén articuladas con su planeación y la implementación de estas deben contribuir a actividades académicas tales como la movilidad, actualización de currículos y la internacionalización de la investigación, con el objetivo de impulsar proyectos que puedan generar nuevos retos competitivos a nivel global.

Palacio y Rondón (2018) en su estudio sobre el marketing relacional, evidencian que el $42.3 \%$ de las instituciones de educación superior en la región caribe de Colombia cuentan con un presupuesto asignado para cubrir los costos de becas de movilidad y descuentos, trabajan en estrategias de gestión internacional, cooperan con universidades internacionales, promueven la movilidad estudiantil de profesores y administrativos y financian proyectos de investigación internacional que fortalecen los currículos y las actividades sustantivas de las instituciones en la docencia, investigación y extensión. A este respecto, De Wit y Hunter (2015) en su modelo de internacionalización muestra como las IES deben incorporar la internacionalización de forma transversal, para esto es necesario desarrollar un análisis interno y externo que permita conocer los beneficios que pueda generar en los asuntos académicos. De la misma manera debe existir un compromiso institucional por parte de los directivos que conlleve a la planificación, operacionalización e implementación de actividades que conduzcan a la revisión y evaluación del proceso de internacionalización.

\section{La gestión de la experiencia}

La gestión de la experiencia es un concepto relativamente nuevo en el marketing, que ha venido evolucionando y teniendo importancia a través del tiempo; limitada en un contexto de servicio enfocado en el comportamiento del consumidor y la experiencia del cliente (Homburg et al., 2017). Estas se ven reflejadas en la dirección estratégica, que tiene como objetivo lograr y mantener la lealtad del cliente a largo plazo, resaltada principalmente en la cultura organizacional de la empresa, siendo esta la llave para algunas compañías de servicios, y la experiencia uno de los puntos de referencias de algunos autores (Gronholdt et al., 2015). La gestión de la experiencia se considera como una de las estrategias que presentan un ganarganar de cambio de valor en las empresas y consumidores. En este contexto, se debe concentrar en la experiencia vivida del servicio del cliente como un conocimiento potencial de la experiencia para ser utilizado por la organización e innovar (Jazir, 2018); reflejando el diseño de marca para construir y satisfacerlas necesidades existentes. 
Verhoef et al. (2009) establecen en su modelo conceptual sobre la creación de la experiencia, es importante tener presente varios aspectos que se resaltan entre esta y sus moderadores; los cuales incluyen, 1) el entorno social; 2) la interfaz de servicios; 3) el ambiente de venta; 4) el surtido; 5) el precio y las promociones. La experiencia se ve afectada por el pasado e incluso determina el consumo y los moderadores en el momento de desarrollar la compra.

Por su parte Hwang y Seo. (2016) consideran que no solamente es co-crear nuevas experiencias, se debe buscar una perspectiva de servicio y las empresas deben verse como facilitadoras (Hulten, 2011). Esto hace referencia a que las empresas deben utilizar interacciones con los clientes para influir en los procesos de la creación del valor. Siendo así, el colaborador como un cliente interno (Payares et al., 2019), de esta manera, las organizaciones deben reflejar motivación, bienestar y compromiso con los empleados, entendiendo que son participantes activos de los procesos rutinarios donde el objetivo es la entrega de servicios que satisfagan al cliente externo (Prieto et al., 2016).

Para comprender la gestión de la experiencia, primero hay que tener claridad de los factores que hacen parte de esta. Abarcando la literatura existente, surge el primer factor, el cual es la experiencia, es considerada como los momentos memorables del cliente. Precursores como Hirschman y Holbrook (1982) han demostrado que, en la experiencia, las emociones son esenciales en dos áreas, primero, en los contextos de elección, los cuales tienen un gran potencial para mejorar la decisión del consumidor y la construcción de experticias multisensoriales, imágenes de fantasías y respuestas que motivan al individuo en diversos procesos de consumo. Schmitt (1999) afirma que el marketing experiencial está en todas partes y esto ocurre con el resultado de tres mejoramientos simultáneos en el entorno empresarial, los cuales son: 1) la presencia de información tecnológica: hoy en día aumenta la tecnología en los procesos de las organizaciones; 2) la supremacía de la marca: trabajada hacia el ciclo de la innovación y 3) La ubicuidad del entrenamiento y las comunicaciones.

Otros precursores como Gilmore y Pine (2002) afirman que las empresas aprenderán como pueden ofrecer al mercado experiencias que enganchen a los clientes potenciales. Por lo tanto, los últimos trabajos realizados en el ámbito de estudio han centrado su interés en el efecto positivo que se deriva de la aplicación de una óptica experiencial del marketing en la lealtad del consumidor (Moral y Fernández, 2012), porque la existencia de productos sustitutos, nuevos modelos de negocios, interdependencia e internacionalización de las empresas, aumentan la oferta de los productos y empiezan a incluir la experiencia como una fuente de valor incrementando en la vinculación personal de su objeto de estudio. La experiencia la consideran intrínsecamente personales, existiendo solo en la mente de un individuo que ha estado involucrado (Stein y Ramaseshan, 2016). Por esta razón, se ha demostrado que las experiencias proporcionan valores cognitivos, emocionales, conductuales y relacionales que reemplazan los valores funcionales Schmitt (2010); se hace referencia a la personalización de cada una de las estrategias que se implementen para generar experiencias, puesto que, en los diseños de los servicios se articulan los cinco sentidos, siendo estos los que permiten que las experiencias sean personales como lo manifestó Gilmore y Pine (2002) en uno de sus aportes.

El segundo factor que se resalta son los proveedores de experiencias, que están más relacionados con los puntos de contactos y son los que producen modelos experienciales, siendo definidos como instrumentos tácticos basados en un conjunto de sensaciones, sentimientos, actuaciones o relaciones, mediante el uso de las comunicaciones, tanto internas como externas en la comunidad académica. Entre los proveedores de experiencia más utilizados están: 1) la publicidad, 2) la identidad visual/verbal corporativa; 3) la presencia del producto; 4) las nuevas marcas; 5) los entornos espaciales y 6) los medios electrónicos que permiten experiencias interactivas hasta el personal de la empresa (Moral y Fernández, 2012). A este respecto, las organizaciones deben elegir muy bien los medios de comunicación que incluirán en su estrategia para lograr atraer a los clientes que conozcan la marca y al mismo tiempo sean referentes. Por otra parte, el papel de la tecnología en las interacciones cliente-empresa y el número de productos y servicios basados en la tecnología ha crecido rápidamente (Parasuraman, 2000), dando como resultado que la tecnología interactúe con el estudiante o profesor y existan sistemas tecnológicos en lugar de personal en las instituciones de educación superior.

Stein y Ramaseshan (2016) mencionan en su estudio que diferentes puntos de contactos ocurren a lo largo del viaje del cliente para mejorar la experiencia, y los hallazgos enfatizan que otros elementos distintos de la experiencia del cliente generan puntos de contacto, e incluyen: 1) los procesos, 2) la interacción entre empleado-cliente y 3) la interacción cliente-cliente. Así mismo, la identificación de los puntos de contactos y su medición debe evaluar la perspectiva de los participantes, porque, al identificarlos, los directores o jefes del proceso deben considerarlo para obtener un enfoque más holístico y comprensión integral de la experiencia.

Sin embargo, para llevar a cabo la estrategia de la gestión de la experiencia, es necesario incluir el tercer factor, el cual es la innovación en el diseño del servicio. La investigación en innovación de servicios amplia el 
estudio de ecosistemas de servicios para hacer un uso creativo y efectivo del papel de plataformas de innovación, mediante la participación con múltiples actores, entendiendo estructuras dinámicas y gestionando el proceso (Bo y Tronvoll, 2020).

Iriarte et al., (2016) mencionan que los retos de las organizaciones se basan en la necesidad de establecer una estructura capaz de diseñar y entregar un sistema de producto y servicio fundamentados en una nueva cultura sobre la generación del valor añadido al valor co-creado y el valor en uso. Por consiguiente, Henrique et al. (2020) argumentan que la innovación puede traer ventajas relacionadas con el negocio, tanto internas como externas, especialmente a través de procesos de producción en la generación de productos y servicios, logrando la eficiencia y creación comercial para la comunidad académica y empleados, derivado de una combinación de tipos de innovación que operan en varios frentes de la estructura organizacional. Así mismo, los diseños en los sistemas facilitan la búsqueda de revisión para la recopilación, análisis interpretación y visualización de datos del cliente (Liu et al., 2019).

En este sentido, el cuarto factor es el proceso de la gestión de la experiencia, el cual articula varios pasos a seguir para administrar estratégicamente las experiencias. Según Schmitt. (2010) presenta una metodología enfocada en 5 pasos, que son: 1) analizar la experiencia generando una constante actualización del cliente a través del tiempo; 2) la construcción de una plataforma que es la conexión entre la estrategia y la implementación a desarrollar; 3) el diseño experiencial de marca, involucrando relación con los elementos visuales y corporativo de las organizaciones con el cliente externo;4)la estructuración de la experiencia logrando un punto de contacto con el cliente externo y 5) la construcción de estrategias relacionadas a la satisfacción y las relaciones con el cliente, siendo el último paso lo que el autor llama "participación en la innovación continua", teniendo las instituciones la capacidad de seguir innovando en sus servicios y atraer a la comunidad académica.

\section{METODOLOGÍA}

Esta investigación tiene un enfoque epistemológico deductivo y un paradigma cuantitativo. El trabajo es de tipo descriptivo, tiene un diseño no experimental, porque no existe manipulación deliberada de las variables de estudio. Al tener un enfoque epistemológico deductivo, se basa en la teoría y de ésta se esgriman juicios designados como hipótesis.

La población de análisis la constituyen las instituciones de educación superior de Barranquilla que se encuentren inscritas y activas en el sistema nacional de información de la educación superior clasificadas en 1) Instituciones Técnicas Profesionales; 2) Instituciones Tecnológicas; 3) Instituciones Universitarias o Escuelas Tecnológicas y 4) Universidades con una jurisdicción privada y pública que cumplan con los criterios de inclusión y exclusión. Entre los criterios de exclusión están las instituciones que dentro de su estructura organizacional no desarrollen las funciones de internacionalización y no cuenten con una oficina o personal especializado que trabaje en el área, así mismo, que en la página web no tengan un espacio visual que permita identificar la misión, visión y las diferentes actividades reflejadas en las estrategias de internacionalización que permita el fortalecimiento de esta. Entre los criterios de inclusión están las IES oficiales o privadas que cuenten con una UPI, un espacio visual en su página web y exista un director encargado de la toma de decisiones en la oficina. Para el análisis se diseñó una encuesta estructurada en base a la revisión de la literatura, diseñado con escala tipo Likert de 5 puntos, donde 1 representa "totalmente en desacuerdo" y 5 "Totalmente de acuerdo". Así mismo, fue sometido a validación por juicio de expertos y su confiabilidad con la fórmula de alfa de Cronbach, bajo la actualización de software estadístico SPSS. Se envió la encuesta a 14 directores de IES en Barranquilla, de las cuales 13 aceptaron ser parte de la investigación durante los meses de febrero y marzo del año 2020.

\section{Técnica estadística para el análisis de datos}

Se usó la técnica estadística de regresión múltiple para calcular la similitud en forma de función matemática, siendo este el modelo adecuado para predecir la gestión de la experiencia a partir de los siguientes factores independientes: 1) proceso de internacionalización;2) experiencia 3) proveedores de experiencia;4) diseño del servicio y 5) proceso de la gestión de la experiencia, que permite examinar la gestión de la experiencia como herramienta de la internacionalización. Este modelo de regresión múltiple se lleva a cabo a partir de la prueba de hipótesis para así conocer sus niveles de significancia. Lo cual permitió la confiabilidad de los resultados.

Con base en los aspectos anteriores, se propone la siguiente hipótesis: $\mathrm{H}^{\circ}$ : Existe normalidad entre los factores o $\mathrm{H}^{1}$ : No existe normalidad entre los factores. Una vez identificadas las hipótesis se llevan a cabo las pruebas estadísticas para fijar su nivel de significancia, seguido del desarrollo de pruebas paramétricas para establecer si cumplen con las condiciones de distribución normal. Teniendo en cuenta que el número de la 
muestra es menor a 50 casos se realizó la prueba de normalidad y se empleó la prueba de Shapiro - Wilk para validar las hipótesis. Otro criterio que se incluyo fue determinar si en los factores de la muestra se pueden utilizar pruebas paramétricas para estudiar la existencia de la homocedasticidad y conocer la correlación existente según su significancia.

Este estudio se validó por uno de los supuestos básicos de independencia de entre los residuos basado en el estadístico Durbin Watson, para saber si cumple con los parámetros de independencia establecidos y se desarrolla el coeficiente de correlación de Pearson. Con el análisis de Anova se evaluó si existía una diferencia significativa entre los grupos de medias, para evidenciar si los resultados obtenidos son constantes en el modelo. Una vez, obtenida la ecuación de regresión de la gestión de la experiencia se utilizó la regresión por bloques para conocer la jerarquía existente entre ellas y calcular la contribución de cada una de ellas según la importancia asignada.

\section{RESULTADOS}

Según los resultados obtenidos de la prueba de Shapiro- Wilk el valor de la significancia de los factores es mayor a 0,05. v. De igual forma, se evidenció que el factor independiente proveedores de experiencia no tiene tanta relevancia porque su significancia es menor e igual a 0.048. Una vez conocida su normalidad, se desarrollaron los diagramas de regresión parcial que permitieron inferir que el factor dependiente, gestión de la experiencia, y los factores independientes diseño de servicio, experiencia y proceso de la gestión de la experiencia, cumplen con el supuesto de normalidad, es decir tienen una distribución normal. Otro indicador que se incluyo fue la tolerancia y el factor de inflación (VIF) de la varianza, este factor mostró que la experiencia, el diseño de servicio y el proceso de la gestión de la experiencia, presentan una alta tolerancia y por tanto una pequeña VIF, lo que indica que no presentan colinealidad.

La prueba del estadístico Durbin-Watson arrojó un valor de 1.892, verificando que los residuos son independientes, por encontrarse entre los rangos de 1.5 y 2.5, es decir que no existe auto correlación entre los factores gestación de la experiencia, diseño de servicio, experiencia y procesos de gestión de la experiencia. Así mismo, al no presentar una estructura definida respecto de los valores predichos por el modelo, no se rechazó la hipótesis de homocedasticidad. Con el coeficiente de correlación de Pearson, se conoce la valoración de la relación existente entre los factores estudiados, teniendo presente que existe mayor correlación si los factores son más cercanos a 1. Según los resultados evidenciados en la Tabla 1.

Tabla 1: Correlación entre los factores independientes.

\begin{tabular}{|c|c|c|c|c|c|c|c|c|}
\hline \multirow{7}{*}{$\begin{array}{c}\mathrm{N}: 13 \\
\text { Correlación } \\
\text { de Pearson }\end{array}$} & \# & Factores & 1 & 2 & 3 & 4 & 5 & 6 \\
\hline & 1 & La gestión de la experiencia & 1 & 0.453 & $0.619^{*}$ & $0.650^{*}$ & $0.866^{* *}$ & $0.771^{\star *}$ \\
\hline & 2 & Proceso de internacionalización & 0.453 & 1 & -0.043 & $0.772^{* *}$ & $0.587^{*}$ & 0.538 \\
\hline & 3 & Experiencia del & $0.619^{*}$ & -0.043 & 1 & 0.234 & 0.409 & 0,277 \\
\hline & 4 & Proveedores de experiencia & $0.650^{*}$ & $0.772^{* *}$ & 0.234 & 1 & $0.761^{* \star}$ & $0.672^{*}$ \\
\hline & 5 & Diseño del servicio & $0.866^{* *}$ & $0.587^{\star}$ & 0.409 & $0.761^{* *}$ & 1 & $0.902^{* *}$ \\
\hline & 6 & Proceso de la Gestión de la experiencia & $0.771^{* *}$ & 0.538 & 0.277 & 0.672 & $0.902^{* *}$ & 1 \\
\hline \multirow{6}{*}{$\begin{array}{c}\text { Sig. } \\
\text { (bilateral) }\end{array}$} & 1 & La gestión de la experiencia & & 0.12 & 0.024 & 0.016 & 0 & 0.002 \\
\hline & 2 & Proceso de internacionalización & 0.12 & & 0.889 & 0.002 & 0.035 & 0.058 \\
\hline & 3 & Experiencia & 0.024 & 0.889 & & 0.443 & 0.166 & 0.36 \\
\hline & 4 & Proveedores de experiencia & 0.016 & 0.002 & 0.443 & & 0.003 & 0.012 \\
\hline & 5 & Diseño del servicio & 0 & 0.035 & 0.166 & 0.003 & & 0 \\
\hline & 6 & Proceso de la Gestión de la experiencia & 0.002 & 0.058 & 0.36 & 0.012 & 0 & \\
\hline
\end{tabular}

La tabla 1 demuestra que la correlación significativa en el nivel 0.05 (bilateral) representada por un asterisco y la significancia en el nivel 0.01 (bilateral) representada por dos asteriscos. De acuerdo con los resultados, la gestión de la experiencia tiene correlación de 0.619 con experiencia del cliente y proveedores de experiencia de 0.650 significativa en el nivel 0.05 ; el diseño de servicio tiene una correlación de 0.866 y proceso de la gestión de la experiencia del cliente en 0.771, con una significativa en el nivel 0.01, lo que demuestra que existe una correlación positiva adecuada entre los factores dependientes e independientes.

El factor independiente proveedores de experiencia tiene una correlación de 0.772 con los factores proceso de internacionalización de 0.650 y diseño de servicio de 0.761 , con una significativa en el nivel 0.01 ; de igual forma el factor independiente diseño de servicio se encuentra correlacionada con el factor gestión de la experiencia del cliente 0.866 , proveedores de experiencia 0.761 y proceso de la gestión de la experiencia de 
0.902 con una significativa en el nivel 0,01 siendo una correlación positiva alta. El factor independiente, proceso de la gestión de la experiencia del cliente se encuentra correlacionado con el diseño del servicio 0.902. Esto indicó que existe un supuesto de colinealidad entre estos factores independientes. Una vez obtenidas las correlaciones con la técnica estadística de regresión múltiple se puede predecir, que los predictores constantes proceso de la gestión de la experiencia, experiencia, proceso de internacionalización, proveedores de experiencia y diseño del servicio son dependientes a la gestión de la experiencia. De acuerdo con lo expresado anteriormente, se desarrolla el resumen del modelo como componente de estudio en la Tabla 2.

Tabla 2: Resumen del modelo según el estadístico Durbin-Watson.

\begin{tabular}{|c|c|c|c|c|c|c|c|c|c|c|}
\hline \multirow[b]{2}{*}{ Modelo } & \multirow[b]{2}{*}{$\mathrm{R}$} & \multirow[b]{2}{*}{$\mathrm{R}^{2}$} & \multirow{2}{*}{$\begin{array}{c}\mathrm{R}^{2} \\
\text { ajustado }\end{array}$} & \multirow{2}{*}{$\begin{array}{c}\text { Error estándar } \\
\text { de la } \\
\text { estimación }\end{array}$} & \multicolumn{5}{|c|}{ Estadísticos de cambio } & \multirow{2}{*}{$\begin{array}{l}\text { Durbin- } \\
\text { Watson }\end{array}$} \\
\hline & & & & & $\begin{array}{l}\text { Cambio en R } \\
\text { cuadrado }\end{array}$ & $\begin{array}{l}\text { Cambio } \\
\text { en F }\end{array}$ & gl1 & gl2 & $\begin{array}{l}\text { Sig. Cambio } \\
\text { en } F\end{array}$ & \\
\hline 1 & 0.916 & 0.839 & 0.724 & 0.2592 & 0.839 & 7.301 & 5 & 7 & 0.011 & 2.088 \\
\hline
\end{tabular}

En la tabla 2 muestra que el coeficiente de correlación lineal es igual a $91.6 \%$, lo que indica que existe buena correlación lineal entre los factores, además, el modelo explica un $83.9 \%$, que una vez corregido por el efecto de la muestra y de los factores independientes resulta el mismo porcentaje $83.9 \%$. Por otro lado, el error típico de la estimación (raíz cuadrada de la varianza no explicada) resulta ser de 0.2592 y el valor del estadístico Durbin Watson es 2.088, es decir que cumple con los parámetros de independencia establecidos. El análisis de la varianza Anova indica que el modelo de regresión es significativo ( $\mathrm{p}$-valor $<0,05$ ). Por tanto, se rechaza la hipótesis nula de que la variabilidad observada en el factor da respuesta que sea explicada por el azar, admitiendo que hay algún tipo de asociación entre el factor dependiente y las independientes.

Se construyó la tabla del modelo de regresión lineal múltiple con los factores independientes (proceso de internacionalización, experiencia, proveedores de experiencia, diseño de servicio y proceso de gestión de la experiencia) para describir la relación con el factor dependiente (gestión de la experiencia), lo cual evidenció que los procesos de internacionalización y proveedores de experiencias no son significativos. Debido a que el valor-P fue mayor a 0.05 , estos factores no son estadísticamente significativos con un nivel de confianza del $95.0 \%$. Por consiguiente, se hace necesario excluir estos dos factores simplificando el modelo solo con las constantes de experiencia del cliente, diseño de servicio y proceso de la gestión de la experiencia del cliente como se refleja en la Tabla 3.

Tabla 3: Modelo de regresión con factores significativos.

\begin{tabular}{|c|c|c|c|c|c|c|c|c|c|}
\hline & \multirow{2}{*}{ Modelo } & \multicolumn{2}{|c|}{$\begin{array}{l}\text { Coeficientes no } \\
\text { estandarizados }\end{array}$} & \multirow{2}{*}{$\begin{array}{c}\begin{array}{c}\text { Coeficientes } \\
\text { estandarizados }\end{array} \\
\text { Beta }\end{array}$} & \multirow{2}{*}{$\mathrm{t}$} & \multirow{2}{*}{ Sig. } & \multicolumn{3}{|c|}{ Estadísticas de colinealidad } \\
\hline & & $\mathrm{B}$ & $\begin{array}{l}\text { Error } \\
\text { estándar }\end{array}$ & & & & $\begin{array}{l}\text { Orden } \\
\text { cero }\end{array}$ & Tolerancia & VIF \\
\hline \multirow[t]{4}{*}{1} & (Constante) & 0.239 & 0.582 & & 0.41 & 0.691 & & & \\
\hline & Experiencia & 0.250 & 0.115 & 0.329 & 2.171 & 0.048 & 0.619 & 0.788 & 1.269 \\
\hline & Diseño del servicio & 0.568 & 0.301 & 0.639 & 1.89 & 0.041 & 0.866 & 0.159 & 6.294 \\
\hline & $\begin{array}{l}\text { Proceso de la } \\
\text { Gestión de la } \\
\text { experiencia }\end{array}$ & 0.080 & 0.247 & 0.103 & 0.322 & 0.045 & 0.771 & 0.176 & 5.678 \\
\hline
\end{tabular}

De acuerdo con la tabla 3 se deduce que la ecuación de regresión, en donde: La gestión de la experiencia = $0.239+0.250$ experiencia +0.568 diseño del servicio +0.080 proceso de la gestión de la experiencia. Una vez obtenida la ecuación de regresión de la variable de estudio, se desarrolló una regresión por bloques con el propósito de conocer el porcentaje o nivel de jerarquía y su contribución en los diferentes bloques. La jerarquía utilizada fue: 1) diseño de servicio; 2) experiencia del cliente y 3) proceso de la gestión de la experiencia del cliente, como se refleja en la Tabla 4. 
Tabla 4. Regresión por bloques de los factores significativos.

\begin{tabular}{|c|c|c|c|c|c|c|c|c|c|c|}
\hline \multirow[b]{2}{*}{ Modelo } & \multirow[b]{2}{*}{$\mathrm{R}$} & \multirow[b]{2}{*}{$\mathrm{R}^{2}$} & \multirow{2}{*}{$\begin{array}{c}\mathrm{R}^{2} \\
\text { ajustado }\end{array}$} & \multirow{2}{*}{$\begin{array}{l}\text { Error } \\
\text { estándar }\end{array}$} & \multicolumn{5}{|c|}{ Estadisticos de cambio } & \multirow{2}{*}{$\begin{array}{l}\text { Durbin- } \\
\text { Watson }\end{array}$} \\
\hline & & & & & $\begin{array}{c}\text { Cambio } \\
\text { en } R^{2}\end{array}$ & Cambio en $\mathrm{F}$ & gl1 & gl2 & $\begin{array}{l}\text { Sig. Cambio } \\
\text { en F }\end{array}$ & \\
\hline 1 & $0.866^{\mathrm{a}}$ & 0.751 & 0.728 & 0.2575 & 0.751 & 33,097 & 1 & 11 & 0.000 & \\
\hline 2 & $0.914^{b}$ & 0.835 & 0.802 & 0.2197 & 0.084 & 5,104 & 1 & 10 & 0.047 & \\
\hline 3 & $0.915^{c}$ & 0.837 & 0.782 & 0.2303 & 0.002 & 0.103 & 1 & 9 & 0.755 & 1.892 \\
\hline
\end{tabular}

La tabla 4 muestra la regresión por bloque se evidencia que la gestión de la experiencia del cliente es el factor que contribuirá en todo su valor, el factor diseño de servicio tiene su contribución del $86.6 \%$ y su significación es 0.000 , la experiencia del cliente tiene una contribución del $91.4 \%$ y su significancia 0.047 y la variable proceso de la gestión de la experiencia del cliente $91.5 \%$ con una significancia de 0.755 .

\section{DISCUSIÓN FINAL}

Los resultados evidencian en primer lugar que de los cinco factores independiente en este estudio solo tres son significativos para la gestión de la experiencia del cliente, siendo los proveedores de experiencia uno de los factores que tiene menor significancia en las IES. Sin embargo, Moral y Fernández (2012) y Holmlund et al. (2020) resaltan su importancia y señalan que los proveedores de experiencias y puntos de contacto como 1) el ambiente; 2) la interacción de la empresa con el cliente y 3) los medios de comunicación producen modelos experienciales basados en las sensaciones, emociones y sentimientos que generan experiencia. El otro factor con menor significancia es el proceso de la internacionalización, esto quiere decir que las UPI no están realmente consientes de la importancia de garantizar que existan experiencias positivas en su comunidad académica derivadas de la planificación de las actividades de internacionalización como 1) la promoción de la movilidad académica; 2) la internacionalización del currículo y 3) las actividades relacionadas con la gestión, investigación y cooperación internacional. De acuerdo con esto, Palacio y Rondón (2018) evidencian que solo el $38.5 \%$ de las IES de las regiones Caribe de Colombia tiene afiliación a redes internacionales con todos sus grupos de interés y solo el 34.6\% de las IES encuestadas manifiestan realizar investigaciones con universidades homologas. En este sentido, el pronóstico de Salmi et al. (2014) sobre la dificultad de Colombia para ubicarse entre los países más competitivos para América Latina en el año 2032 se hace evidente.

A su vez, este estudio destaca que la gestión de la experiencia se convierte en una herramienta para la internacionalización de la educación superior, puesto que la experiencia, es el factor que tiene una mayor correlación con ella, lo que significa que las UPI deben construir estrategias relacionadas principalmente con vivencias, incluyendo las emociones, sensaciones, percepciones y motivaciones como lo determina el autor Schmitt (2010) cuando expresa que un proceso sistemático va desde el conocimiento de las expectativas del cliente hasta la medición por indicadores del proceso, seguido del diseño del servicio. Además, sugiere mapear los puntos de contacto del cliente interno con el cliente externo para desarrollar mejoras que se reflejen en avances tecnológicos. Así mismo, Iriarte et al. (2016) afirman que se debe aportar valor a los procesos desde la óptica del usuario y que se incluyan protocolos de atención e indicadores que proporcionen medición de las actividades relacionadas con el servicio ofrecido por las UPI.

Así mismo, el factor independiente diseño del servicio tiene una correlación positiva alta con la experiencia y con el proceso de la gestión de la experiencia siendo un aporte significativo al modelo de Schmitt. (2003). De ahí que, la incorporación de las tecnologías en los procesos del servicio ofrecido debe estar basadas en la creación de momentos memorables. Para ello, es necesario hacer investigaciones de mercado para analizar las preferencias y necesidades del consumidor y así identificar los vínculos y afinidades del cliente a través de herramientas como software especializados en el servicio. De esta manera las guías de procedimiento de atención y la comunicación con el cliente interno por medio de encuestas de satisfacción o protocolos del proceso ayudan a identificar la percepción de la marca, y así se pueden diseñar experiencias del cliente que estén enfocadas en su fidelización y satisfacción de los servicios.

\section{CONCLUSIONES}

De acuerdo con los resultados de este estudio, la gestión de la experiencia constituye una herramienta para la internacionalización de las IES de Barranquilla, cuando se incorpora en el proceso de atención estrategias de marketing experiencial que en los puntos de contacto den a conocer vivencias de internacionalización que activen las emociones, sensaciones y percepciones, logrando experiencias enriquecedoras y animen a participar a la comunidad académica en los servicios ofrecidos en las unidades principales de 
internacionalización. De la misma manera la construcción de plataformas que generen conexión entre las estrategias y su implementación son una de las claves para diseñar experiencias de marca en donde se deben involucrar los elementos visuales corporativos de la UPI con el cliente externo.

Con la ecuación de la técnica estadística de regresión múltiple de la gestión de la experiencia se logra identificar los niveles de importancia de factores independientes como lo son: el diseño de servicios, la experiencia del cliente y el proceso de la gestión de la experiencia del cliente. Los factores independientes utilizados en los servicios públicos de interés traerán consigo esfuerzos para la percepción de marca y la utilización de herramientas tecnológicas para la innovación continua.

Por consiguiente, con la adquisición de software especializado para gestionar el servicio, y la construcción de protocolos de atención para manejar las objeciones que se presentan, se podrá monitorear de manera permanente las interacciones que generan experiencias por medio de indicadores que evalúen la calidad del servicio. Así mismo, esta estrategia logra tener interacción con los diferentes niveles de acción, aumentando las expectativas y generando lealtad de marca en los diferentes ámbitos de estudio, siendo parte principal de la internacionalización.

\section{REFERENCIAS}

Aguilar, Y. y Riveros, A. S., La internacionalización de la educación superior: Concepto evolución del modelo en la Universidad de Costa Rica, https://doi.10.15517/revedu.v4111.17952, Revista educación, 41(1),73-103 (2016)

Andajani, E., Understanding Customer Experience Management in Retailing, https://doi.10.1016/j.sbspro.2015.11.082 Procedia - Social and Behavioral Sciences, 211,629-633 (2015)

Bo, E. y Tronvoll , B., A new conceptualization of service innovation grounded in S-D logic and service systems, https://doi.10.1108/17566691311316220, International Journal of Quality and Service Sciences, 5(1), 19-31 (2020)

De keiser, A., Verleye, K., Lemon, K. N., Keiningham, T.L.y Klaus, P., Moving the customer experience field forward: introducing the touchpoints, context, qualities (TCQ) nomenclature, https://doi.org/10.1177/1094670520928390, Journal of Service Research, 1, 1-23 (2020)

De Wit, H. y F. Hunter, El futuro de la internacionalización de la educación superior en Europa, International Higher Education, 83, 2-3 (2015)

Geetika, V, Gopal Das, A. y Arpita K., Experiential value: a review and future research directions, https://doi. 10.1108/MIP05-2016-0075, Marketing intelligence and planning,35, 21, 339-357 (2017)

Gronholdt, L, Martensen, A, Jorgensen, S. y Jensen P, Customer experience management and business performance, https://doi.org/10.1108/lJQSS-01-2015-0008,International Journal of Quality and Service Sciences, 7(1), 90-106 (2015)

Henrique, T. F., Lopez, M.L., Basso, L.F., Areal, N., Indicators used to measure service innovation and manufacturing innovation, https://doi.org/10.1016/j.jik.2019.12.001, Journal of innovation and Knowledge, 12(3),1-16 (2020)

Hirschman, E. C. y M. B Holbrook, Hedonic Consumption: Emerging Concepts, Methods and Propositions, https://:doi.10.1177/002224298204600314, Journal of Marketing, 46(3), 92-101(1982)

Holmlund, M, Van, V.Y, Ciuchita, R, Ravald, A, Sarantopoulos, P, Villarroel, O.F. y Zaky,M., Customer experience management in the age of big data analytic: A strategic framework, https://doi.org/10.1016/j.jbusres.2020.01.022 Journal of Business Research,1, 1-10 (2020)

Homburg, C, Jozić, D. y Kuehnl C., Customer experience management: toward implementing an evolving marketing concept, https://doi.org/10.1007/s11747-015-0460-7, Journal of the Academy of Marketing Science, 45(3), 377-401 (2017)

Huerta, P.C., y Gaete, H.G. Convenios Marco y su Aplicación en las Universidades Públicas Chilenas: Periodo 2015-2016, http://dx.doi.org/10.4067/S0718-50062019000200105, Form. Univ., v.12, n. 2, p. 105-120 (2019)

Hultén, B., "Sensory Marketing: The Multi-Sensory Brand-Experience Concept", , https://doi.10.1108/09555341111130245, European Business Review, 23, 3, 256-273 (2011)

Hwang, J., y S. Seo, A critical review of research on customer experience management: Theoretical, methodological and cultural perspectives, https://doi.org/10.1108/IJCHM-04-2015-0192, International Journal of Contemporary Hospitality Management, 28(10), 2218-2246 (2016)

Iriarte, I, Justel, D, Alberdi, A, Val, E. y Gonzalez, I. Diseño de servicios para la servitización. Experiencias con empresas manufactureras vascas a través de la colaboración universidad-empresa. Universia Business Review, ISSN: 1698-5117 49,146-181 (2016)

Jaziri, D., The advent of customer experiential knowledge management approach (CEKM): The integration of offline \& online experiential knowledge, https:// doi.org/10.1016/j.jbusres.2018.05.029, Journal of Business Research, 94, 241-256 (2018) 
Liu, Y., Soroka, A., Han, L., Jian, J., y Tang, M., Cloud-based big data analytics for customer insight-driven design innovation in SMEs. https://doi.10.1016/j.jinfomgt.2019.11.002.,51, 102034 (2020)

Ministerio de Educación, Gestión de la Internacionalización - Guías para la internacionalización de la educación superior, Challenge Your Knowledge, $1^{\circ}$ Ed, 1,1-47 (2015)

Moral, M.y Fernández M., Nuevas Tendencias del Marketing: El Marketing Experiencial, revista Interdisciplinar, ISSN-e 1885-698514,237-251,(2012)

Ortega, B.J.A., Tensión educativa entre la tradición y las nuevas alternativas, Revista Cubana de Educación Médica Superior, 34(1), 1-12, (2020)

Palacio, S. y C. Rondón, Marketing relacional para la Internacionalización en Institución de Educación Superior. Revista Espacios, ISSN 0798 1015, 39, 23, 1-15, (2018)

Parasuraman, A., Technology Readiness Index (Tri): A Multiple-Item Scale to Measure Readiness to Embrace New Technologies, https:// doi.org/10.1177/109467050024001, Journal of Service Research,2(4), 307-320 (2000)

Payares, K. M, Parra, M, Navarro, E.J. y Naranjo, O, Mercadeo interno en las pequeñas y medianas empresas del sector salud de Barranquilla(Colombia),https://doi.org/10.4067/S0718-07642020000100123, Cit información tecnologica,31(1), 123-132 (2020)

Gilmore, J.H y B.J Pine, Customer experience place: the new offering fronter, https://doi.10.1108/10878570210435306, Strategy and Leadership, 30,4,4-11(2002)

Prieto, R, Burgos, C, García, J. y Rincón, Y, Mercadeo interno para optimizar la calidad de servicio en la banca universal, https://doi.10.31876/revista.v21i73.21059 Revista Venezolana de Gerencia, 21(73), 102-119 (2016)

Samil.J, Martínez, P, Nupia C. M. y otros 17 autores, Política y gestión de la internacionalización de la educación superior en Colombia. Reflexiones para la Política de Internacionalización de la Eduación Superior en Colombia, $1^{\circ}$ Ed., Observatorio Colombiano de Ciencia y Tecnología (OCyT), 1, 77-99 (2014)

Schmitt, B. H, The customer experience management: A revolutionary approach to connecting with your customers, $1^{\circ}$ Ed.,288, John Wiley \& Sons Limited, New Jersey, Estados Unidos (2010)

Schmitt, B., Experiential marketing, https://doi.org/10.1362/026725799784870496, Journal of Marketing Management, 15(1-3),53-67 (1999)

Stein, A.y B.Ramaseshan,Towards the identification of customer experience touch point elements, https://doi.org/10.1016/j.jretconser.2015.12.001,Journal of Retailing and Consumer Services, 30, 8-19( 2016)

Verhoef, P. C., Lemon K. N,Parasuraman, A. y otros 3 autores, Customer Experience Creation: Determinants, Dynamics and Management Strategies, https://doi.10.1016/j.jretai.2008.11.001, Journal of Retailing, 85(1),31-41( 2009) 